\section{Admission rates for emergency department patients with venous thromboembolism and estimation of the proportion of low risk pulmonary embolism patients: a US perspective}

\author{
Adam J. Singer ${ }^{1}$, Henry C. Thode Jr' ${ }^{1}$ W. Frank Peacock IV ${ }^{2}$ \\ 'Department of Emergency Medicine, Stony Brook University, Stony Brook, NY, USA \\ ${ }^{2}$ Department of Emergency Medicine, Baylor College of Medicine, Houston, TX, USA
}

Objective Introduction of target specific anticoagulants and recent guidelines encourage outpatient management of low risk patients with venous thromboembolism. We describe hospital admission rates over time for patients presenting to US emergency departments (EDs) with deep vein thrombosis (DVT) and pulmonary embolism (PE) and estimate the proportion of low-risk PE patients who could potentially be managed as outpatients.

Methods We performed a structured analysis of the National Hospital Ambulatory Medical Care Survey (a nationally representative weighted sampling of US ED visits) database for the years 2006-2010 including all adult patients with a primary diagnosis of DVT or PE. Simplified pulmonary embolus scoring index (SPESI) scores were determined in patients with PE to identify low risk patients.

Results There were an estimated 652,000 and 394,000 ED visits for DVT and PE over the 5-year period (0.17\%). Mean (SE) age was 59 (1.3), 50\% were female, and $40 \%$ were $>65$ years. Admission rates for DVT and PE were 52\% and 90\% respectively with no significant changes over time. In patients with DVT, predictors for admission were age (odds ratio, 1.03 per year of age [95\% confidence interval, 1.01 to 1.05]) and race (odds ratio, 4.1 [95\% confidence interval, 0.9 to 19.8] for Hispanics and 2.9 [1.2 to 7.4] for Blacks). Of all ED patients with PE, 51\% were low risk based on sPESI scores.

Conclusion Admission rates for DVT and PE have remained high and unchanged, especially with $P E$, minorities, and in older patients. Based on sPESI scores, up to half of PE patients might be eligible for early discharge or outpatient therapy.

Keywords Pulmonary embolism; Low-risk; Simplified pulmonary embolus scoring index score; Outpatients; Emergency service, hospital
elSSN: 2383-4625

Received: 1 March 2016

Revised: 2 May 2016

Accepted: 3 May 2016

Correspondence to: Adam J. Singer Department of Emergency Medicine, Stony Brook University, HSC L4-080 8350 SUNY,

Stony Brook 11794-8300, NY, USA

E-mail: adam.singer@stonybrookmedicine.edu

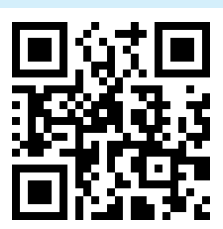

How to cite this article:

Singer AJ, Thode HC Jr, Peacock WF. Admission rates for emergency department patients with venous thromboembolism and estimation of the proportion of low risk pulmonary embolism patients: a US perspective. Clin Exp Emerg Med 2016;3(3):126-131.
This is an Open Access article distributed under the terms of the Creative Commons Attribution Non-Commercial License (http:// creativecommons.org/licenses/by-nc/4.0/). 


Capsule
Summary is already known
$\begin{aligned} & \text { Venous thromboembolism is common and potentially fatal, however low risk patients with deep vein thrombosis and } \\ & \text { pulmonary embolism have a low rate of adverse events and can be managed as outpatients. Introduction of the novel } \\ & \text { oral anticoagulants has made it easier than ever to discharge low risk patients from the emergency department. }\end{aligned}$
$\begin{aligned} & \text { What is new in the current study } \\ & \text { Most patients with pulmonary embolism seen in US emergency departments are admitted to the hospital even though } \\ & \text { up to half may be at low risk for adverse events based on the simplified pulmonary embolus scoring index score and } \\ & \text { could potentially be eligible for outpatient management. }\end{aligned}$

\section{INTRODUCTION}

Venous thromboembolism (VTE) is a disease spectrum ranging from asymptomatic deep vein thrombosis (DVT) to fatal pulmonary embolism (PE). Each year there are approximately 1 million cases of VTE and over 500,000 hospital admissions in the United States with estimated annual costs exceeding 10 billion dollars. ${ }^{1-6}$ According to the Centers for Disease Control and Prevention, VTE kills more people each year than breast cancer, human immunodeficiency virus, and traffic accidents combined. ${ }^{4,5}$ Given the potential for a fatal outcome and limited therapeutic options, patients with VTE have traditionally been admitted to the hospital and started on bridging therapy with a parenteral heparin (either low molecular weight or unfractionated) followed by the oral vitamin K (VKA) antagonist warfarin. However, there is growing evidence that selected patients with VTE, who are at low risk of recurrence or bleeding, can be safely managed as outpatients. Several single and multicenter randomized trials have compared inpatient and outpatient management of DVT using standard care with unfractionated (UH) or low molecular weight heparin (LMWH) followed by traditional oral anticoagulants. ${ }^{7-10}$ These studies have demonstrated that outpatient management using a fixed daily dose of LMWH followed by oral anticoagulation is at least as effective and well tolerated as in the inpatient setting. While the evidence supporting early discharge or outpatient management of low risk PE patients is less robust, it is mounting. A study of patients with low-risk PE (based on pulmonary embolism severity index scores) who were randomized to receive outpatient or inpatient treatment with enoxaparin followed by an oral VKA demonstrated that outpatient care was as safe and effective as inpatient management. ${ }^{11} \mathrm{~A}$ systematic review and meta-analysis also concluded that the rate of adverse events associated with outpatient management of PE was low (the 30 day mortality rate was $1.58 \%$ [95\% confidence interval, $0.71 \%$ to $2.80 \%]$ ]. ${ }^{12}$ Most recently, a study by Beam et al. ${ }^{13}$ demonstrated the safety of a strat- egy of immediate discharge and home treatment with rivaroxaban of low risk VTE patients in two US emergency departments.

As a result of the above evidence, recent guidelines issued by the American College of Chest Physicians (ACCP) encourage outpatient management of low risk DVT patients and early discharge of low risk PE patients. ${ }^{14}$ While outpatient treatment of DVT has become more acceptable in the US, many physicians still have concerns regarding outpatient management or early discharge of low risk PE patients. Identification of patients with PE at low risk of complications has been simplified with the development and validation of scoring systems such as the pulmonary embolism scoring index (PESI) and the simplified pulmonary embolism scoring index (sPESI). ${ }^{11,15}$

The outpatient approach to low risk DVT and PE management has recently become more attractive. This is because the newer target specific oral anticoagulants have a rapid onset precluding the need for parenteral bridging therapy (e.g., rivaroxaban, apixaban), do not require routine anticoagulation testing, have fewer drug-drug interactions, and have no food-drug interactions typical of warfarin (e.g., rivaroxaban, apixaban, dabigatran, edoxaban). ${ }^{16-21}$ Additionally, changes in the healthcare system and financial constraints are leading to a decrease in the number of patients admitted, especially those admitted for short periods.

The goals of the current study were to compare admission rates of US ED patients presenting with PE and DVT over time and to explore the association between predictor variables and hospital admission. We also estimated the proportion of low-risk ED patients with PE who might be eligible for outpatient management or early discharge.

\section{METHODS}

\section{Study design}

We conducted a retrospective analysis of data previously collected by the National Hospital Ambulatory Medical Care Survey (NH- 
AMCS). ${ }^{22,23}$ The study was exempt from institutional review board since the data are available for public access.

\section{Patients and source of data}

NHAMCS is part of the ambulatory component of the National Health Care Survey that measures health care use across various types of providers and was inaugurated in 1992 to gather and disseminate information about the health care provided by hospital EDs to the population of the US. This national probability sample survey, conducted by the Division of Health Care Statistics of the National Center for Health Statistics, Centers for Disease Control and Prevention, samples the target universe of in-person visits made in the US to EDs of non-Federal hospitals. The sampling frame consists of hospitals listed in the April 1991 Specialty Medical Group Hospital Database. Hospital weights are applied to make national estimates. Each sampled hospital provides records for a randomly assigned 4-week reporting period. Data abstraction was performed by local hospital staff who were trained by experts from the US Census Bureau using a standardized abstraction form. The abstractors examined all documents in the medical record, including triage sheets, physician history and physical examination results, nursing records, and physician orders. The abstracted data could include up to 3 possible patient reasons for the visit, 3 physician diagnoses, and cause or causes of injury, as well as a list of all procedures performed and medications ordered or continued. For this analysis, DVT and PE cases were identified using the physician primary diagnoses. The NHAMCS uses the International Classification of Diseases, ninth revision, Clinical Modification, to code the diagnoses. A DVT or PE case for this study was defined as any physician diagnosis citing codes 453.4, 453.41, $453.42,453.5,453.6$ (DVT) or 415.11, 415.19, 416.2 (PE) of the International Classification of Diseases, 9th revision, Clinical Modification, 2.3.

The NHAMCS databases for the years 2006 to 2010 were downloaded from the National Center for Health Statistics web site. Data collected included patient demographics; mode of arrival; time, day, and month of arrival; insurance status; medication prescriptions; practitioner type, and disposition. To calculate incidence rates of DVT and PE, we obtained estimates of the population from the US Census Bureau.

\section{Measures and outcomes}

The primary outcomes were hospital and intensive care unit admission. Secondary outcomes were procedures, medications administered in the ED, sPESI scores and mortality during the ED visit. The SPESI is a clinical prognostic model based on data from nearly 4,000 patients with suspected PE of whom over 1,000 had confirmed PE. ${ }^{15}$ Any score of 1 or more is considered high risk. In the validation study the prognostic accuracy of the original and simplified PESI did not differ. ${ }^{15}$ Patients classified as low risk by the sPESI score had a 30-day mortality of 1.1\% (95\% confidence interval, $0.7 \%$ to $1.5 \%$ ) compared with $10.9 \%$ (95\% confidence interval, $8.5 \%$ to $13.2 \%$ ) in the high-risk group.

\section{Data analysis}

Data were analyzed with IBM SPSS Statistics ver. 22.0 (IBM Corp., Armonk, NY, USA). Examined elements included demographic information, initial vital signs, provider information, insurance status, procedures performed, medications prescribed while in the $E D$, and follow-up information. National estimates were obtained using the assigned NHAMCS patient visit weights.

Patients were classified into two groups based on their presenting diagnosis; DVT or PE. Patients with PE were further classified based on their sPESI scores. Descriptive statistics were calculated, and group comparisons were performed using univariate $\left(\chi^{2}\right.$ and analysis of variance) and multivariate (logistic regression for binary data or linear regression for continuous data) analyses to determine associations of age, gender, race, medications prescribed, and sPESI scores with admission. Admission rates over time were evaluated using unweighted linear regression with the rates as the dependent variable and year as the independent variable.

\section{RESULTS}

There were an estimated 652,000 and 394,000 ED visits for DVT and $\mathrm{PE}$ out of 625 million visits over the 5 -year period $(0.17 \%)$. ED visit rates per 1,000 population were 2.1 and 1.3 for DVT and $\mathrm{PE}$, respectively. Overall, $50 \%$ of patients were female, $72 \%$ were non-Hispanic white, 20\% were non-Hispanic black, and 7\% were Hispanic. Mean (SE) age was 59 (1.3) years, and 40\% were over age 65 . A summary of demographic characteristics by type of VTE is presented in Table 1. Admission rates for DVT and PE were $52 \%$

Table 1. Patient characteristics

\begin{tabular}{lcc}
\hline Characteristics & Deep vein thrombosis & Pulmonary embolism \\
\hline Female & $326,989(52)$ & $192,667(49)$ \\
Mean age (SE) & $58(1.8)$ & $61(2.0)$ \\
Race/ethnicity & & \\
Non-Hispanic white & $466,540(72)$ & $290,119(74)$ \\
Non-Hispanic black & $126,593(19)$ & $79,749(20)$ \\
Hispanic & $57,764(9)$ & $20,614(5)$ \\
Other & $1,363(<1)$ & $3,546(1)$ \\
Admitted & $335,873(52)$ & $355,452(90)$ \\
Intensive care unit & $9,589(2)$ & $61,508(16)$ \\
Died in emergency department & $0(0)$ & $15,001(4)$ \\
\hline
\end{tabular}

Values are presented as number (\%) unless otherwise indicated. 


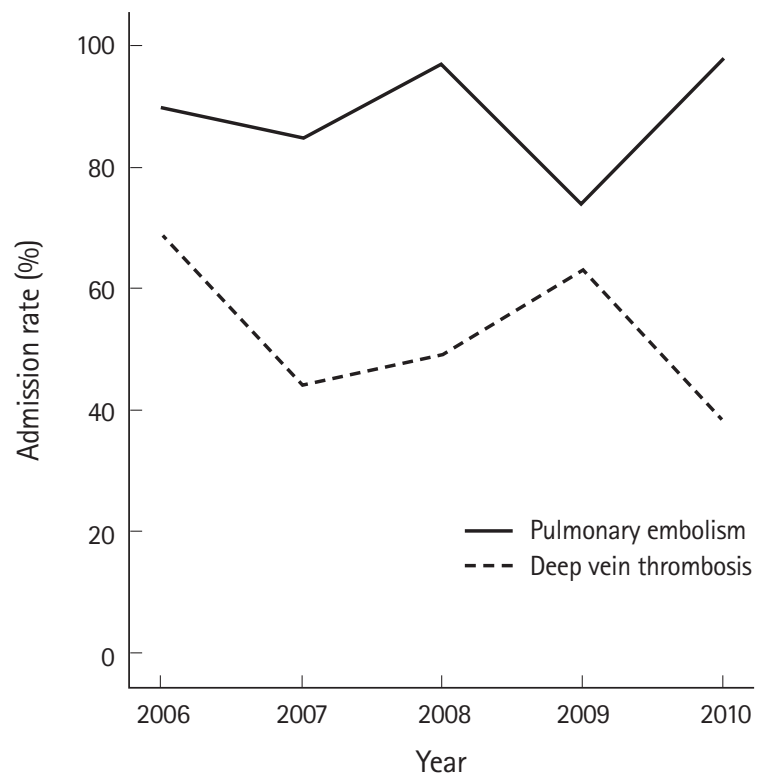

Fig. 1. Trends in hospital admission rates over time.

and 90\% respectively, with no significant trends for either over time (Fig. 1). Intensive care unit admission rates for DVT and PE were $2 \%$ and $16 \%$, respectively.

Of all PE patients, 21\% presented with a heart rate greater than 110 and $4 \%$ presented with a systolic blood pressure of less than $100 \mathrm{mmHg}$. Although not included in the sPESI score, fever (defined as a triage temperature of greater than 37.9 degrees Celsius), was present at a rate of $2 \%$ in each of the PE and DVT cohorts. Hypoxia, defined as a pulse oximetry of less than $94 \%$, was present in $20 \%$ of PE patients. Of all PE patients, 51\% were considered low-risk based on SPESI scores. There was no significant trend in low-risk sPESI patients over time. Of PE patients who were admitted, 54\% were considered as low risk. PE patients were more likely to be admitted in hospitals which were in a Metropolitan Statistical Area (MSA) than in hospitals in non-MSA areas (95\% vs. $56 \%, \mathrm{P}=0.002$ ).

Anticoagulant medications prescribed to patients with DVT and PE are presented in Table 2. Patients with PE were more than 4 times more likely to receive unfractionated heparin than patients with DVT (odd ratio, 4.4; 95\% confidence interval, 1.8 to 11.0 ).

In patients with DVT, predictors for admission were age (odd ratio 1.03 per year [95\% confidence interval, 1.01 to 1.05$]$ ) and race (odd ratio 4.1 [95\% confidence interval 0.9 to 19.8] for Hispanics and 2.9 [1.2 to 7.4] for Blacks). In patients with PE the number of non-admissions was too small to perform multivariate analysis with more than one predictor. Other than MSA (described above), only systolic blood pressure was predictive of admission (odd ratio, 1.05 per unit mmHg; 95\% confidence interval, 1.01 to
Table 2. Anticoagulants prescribed while in emergency department

\begin{tabular}{lcc}
\hline Anticoagulant & Deep vein thrombosis & Pulmonary embolism \\
\hline Fondaparinux & $1,802(0.3)$ & $2,208(0.6)$ \\
Warfarin & $136,725(21)$ & $39,825(10)$ \\
Enoxaparin & $299,125(46)$ & $136,535(35)$ \\
Heparin & $83,374(13)$ & $139,228(35)$ \\
\hline
\end{tabular}

Values are presented as number (\%).

1.10). The sample size was too small to identify predictors of admission in low risk PE patients.

\section{DISCUSSION}

Our results, which are based on a large nationally representative administrative database, demonstrate that nearly half of all DVT patients and the majority of PE patients seen in US emergency departments are admitted to the hospital. Furthermore, these admission rates did not change substantially during the 5 years from 2006 to 2010. Thus, despite mounting evidence and guidelines suggesting that outpatient management of both DVT and low risk PE is safe, it appears that, at least through 2010, discharge from a US ED was unlikely. Despite the limitations of a retrospective database and the lack of information regarding social support systems and bleeding risk, our study suggests that a large number of ED patients with PE (as many as half) are at low risk of adverse events, and could potentially be considered for outpatient management either directly from the ED or after a brief observation period in the ED or inpatient unit. Other studies have also estimated that fifty percent of patients with PE could be treated as outpatients. ${ }^{24}$

It is not surprising that increasing age was associated with admission, since these patients tend to have more co-morbidities and thus be at higher risk. However, it is unclear why minorities, such as Hispanics and blacks, were more likely to be admitted than whites, independent of sPESI scores. It is possible that minorities were less likely to have regular medical care or strong social support systems, which may have led physicians to admit patients despite their being otherwise at low risk of adverse events.

While our analysis is fairly current, the novel anticoagulants that have been approved for both DVT and PE were introduced after the study period. Furthermore, the ACCP guidelines for the management of patients with VTE recommending outpatient management and early discharge were only released in 2012. Thus it is not clear what impact, if any, they may have made on the admission rates of ED patients with VTE. Our study found very low rates of ED discharge for patients with PE. Despite the fact that most patients with PE in the US are admitted to the hospital, there 
is evidence that early discharge of low risk patients is safe. ${ }^{11-13}$

Routine admission of patients with VTE results in a large economic burden. ${ }^{3}$ A study based on a large, nationally representative database including 25 million US patients found that the average costs for hospitalizing a single patient with DVT or PE were 9,805 and 14,106 US dollars respectively. The higher costs associated with hospitalization of PE patients were likely due to a longer hospital length of stay (7.0 vs. 5.6 days for PE vs. DVT, respectively) as well as increased use of inpatient professional services. ${ }^{3}$ Based on our study and assuming that 5 of 10 patients with PE are at low risk of adverse outcomes and could be considered for early discharge or outpatient management, the potential estimated savings exceed 2 billion US dollars $(394,030 \times 50 \% \times 14,106$ US dollars) over the 5 years study period.

The fact that many ED physicians are unaware of, or do not follow, the ACCP guidelines for the management of VTE are supported by a recent study by Pollack et al. ${ }^{25}$ Results from EMPEROR (Multi-center Emergency Pulmonary Embolism in the Real World Registry), a study of the characteristics, outcomes, and management of patients with acute PE in the ED, showed that systemic non-vitamin $\mathrm{K}$-dependent anticoagulation is initiated in the ED in $84 \%$ of patients with VTE. The most common ED treatment was UFH, followed closely by enoxaparin. Only a minority of patients received fondaparinux or dalteparin. Despite recommendations, heparin was administered before diagnostic imaging in only $9 \%$ of patients. While mortality rates for patients diagnosed with PE in US EDs were only 1.1\%, these results still suggest that current treatment guidelines have not been fully integrated into all ED practices. Greater efforts should be made to educate ED practitioners regarding the safety of outpatient management and early discharge of patients with low risk VTE, especially in the era of the target specific oral anticoagulants.

The major limitation of this study is the fact that it is a retrospective analysis of a prospectively collected data set and thus renders our conclusions as "hypothesis generating." In particular calculation of the sPESI scores may be inaccurate since coding of comorbidities, such as heart failure and chronic obstructive pulmonary disease, may not be reliable. Furthermore, data used for estimating sPESI scores was sometimes missing, which likely led to an overestimation of the number of patients at low risk. Of all PE sample patients $14 \%$ had one or more missing values with which to calculate sPESI scores; however, most of those were classified as high risk given the data that was available so that only $3 \%$ of patients may have been misclassified as low risk. Furthermore, our findings that roughly half of all PE patients are low risk is supported by similar findings in other studies. ${ }^{24}$ In addition, the NHAMCS database does not contain any information regard- ing the patients' social support systems, which must be considered when deciding whether it is safe to discharge a patient with VTE, leading to further overestimation of patients eligible for early discharge or outpatient management. Despite these limitations it is important to note that the NHAMCS is a highly recognized and representative federally sponsored survey that lends access to a highly divergent patient population.

Our decision to include patients who were assigned by physicians a diagnosis of VTE as their primary diagnoses is another limitation of the study. Thus, we did not include patients who may have had multiple other medical problems that also had co-existent VTE. In this light, the agreement between the ED physician and discharge diagnoses may have been limited. Despite the fact that the estimated total numbers of patients in the NHAMCS database is very large, data analysis is limited to a relatively small number of actual patients included in the cohort over a 5-year study limiting the power to draw conclusions.

Another limitation of our study is the fact that we could not assess bleeding risk of study patients. While patients with low risk sPESI scores may be considered for early discharge, patients at high risk of bleeding should also be admitted for more prolonged observation. Finally, we only examined the initial vital signs at the time of patient triage. Since vital signs often change over time, we cannot verify that assignment of sPESI scores would be the same later in a patient's presentation. Furthermore laboratory and echocardiographic findings would have been helpful in interpreting our data.

The rates of hospital admission for patients with DVT and PE have remained high and unchanged, especially in patients with $P E$, and in minorities and older patients. Based on estimated SPESI scores, up to half of all PE patients may be eligible for consideration of early discharge or outpatient management, which could lead to significant cost savings and greater patient satisfaction.

\section{CONFLICT OF INTEREST}

No potential conflict of interest relevant to this article was reported.

\section{REFERENCES}

1. Centers for Disease Control and Prevention. Venous thromboembolism in adult hospitalizations: United States, 2007-2009. MMWR Morb Mortal Wkly Rep 2012;61:401-4.

2. Stein PD, Matta F. Epidemiology and incidence: the scope of the problem and risk factors for development of venous thromboembolism. Clin Chest Med 2010;31:611-28. 
3. Spyropoulos AC, Lin J. Direct medical costs of venous thromboembolism and subsequent hospital readmission rates: an administrative claims analysis from 30 managed care organizations. J Manag Care Pharm 2007;13:475-86.

4. Murphy SL, Kochanek KD, Xu J, Heron M. Deaths: final data for 2012 [Internet]. Atlanta, GA: Centers for Disease Control and Prevention; 2015 [cited 2016 Feb 1]. Available from: http: //www.cdc.gov/nchs/data/nvsr/nvsr63/nvsr63_09.pdf.

5. Office of the Surgeon General. The Surgeon General's call to action to prevent deep vein thrombosis and pulmonary embolism [Internet]. Bethesda, MD: National Center for Biotechnology Information; 2008 [cited 2016 Feb 1]. Available from: http://www.ncbi.nlm.nih.gov/books/NBK44178/.

6. Heit JA. The epidemiology of venous thromboembolism in the community. Arterioscler Thromb Vasc Biol 2008;28:370-2.

7. Ramacciotti $E_{1}$ Araujo GR, Lastoria $S$, et al. An open-label, comparative study of the efficacy and safety of once-daily dose of enoxaparin versus unfractionated heparin in the treatment of proximal lower limb deep-vein thrombosis. Thromb Res 2004; 114:149-53.

8. Daskalopoulos ME, Daskalopoulou SS, Tzortzis E, et al. Longterm treatment of deep venous thrombosis with a low molecular weight heparin (tinzaparin): a prospective randomized trial. Eur J Vasc Endovasc Surg 2005;29:638-50.

9. Chong BH, Brighton TA, Baker RI, Thurlow $\mathrm{P}$, Lee $\mathrm{CH}$; ASTH DVT Study Group. Once-daily enoxaparin in the outpatient setting versus unfractionated heparin in hospital for the treatment of symptomatic deep-vein thrombosis. J Thromb Thrombolysis 2005;19:173-81.

10. Boccalon H, Elias A, Chale JJ, Cadene A, Gabriel S. Clinical outcome and cost of hospital vs home treatment of proximal deep vein thrombosis with a low-molecular-weight heparin: the Vascular Midi-Pyrenees study. Arch Intern Med 2000;160:176973.

11. Aujesky D, Roy PM, Verschuren F, et al. Outpatient versus inpatient treatment for patients with acute pulmonary embolism: an international, open-label, randomised, non-inferiority trial. Lancet 2011;378:41-8.

12. Piran S, Le Gal G, Wells PS, et al. Outpatient treatment of symptomatic pulmonary embolism: a systematic review and metaanalysis. Thromb Res 2013;132:515-9.

13. Beam DM, Kahler ZP, Kline JA. Immediate discharge and home treatment with rivaroxaban of low-risk venous thromboem- bolism diagnosed in two U.S. emergency departments: a oneyear preplanned analysis. Acad Emerg Med 2015;22:788-95.

14. Kearon C, Akl EA, Comerota AJ, et al. Antithrombotic therapy for VTE disease: Antithrombotic Therapy and Prevention of Thrombosis, 9th ed: American College of Chest Physicians Evidence-Based Clinical Practice Guidelines. Chest 2012;141(2 Suppl):e419S-94S.

15. Jimenez D, Aujesky D, Moores $L$, et al. Simplification of the pulmonary embolism severity index for prognostication in patients with acute symptomatic pulmonary embolism. Arch Intern Med 2010;170:1383-9.

16. EINSTEIN Investigators, Bauersachs $R$, Berkowitz SD, et al. Oral rivaroxaban for symptomatic venous thromboembolism. N Engl J Med 2010;363:2499-510.

17. EINSTEIN-PE Investigators, Buller HR, Prins MH, et al. Oral rivaroxaban for the treatment of symptomatic pulmonary embolism. N Engl J Med 2012;366:1287-97.

18. Schulman S, Kearon C, Kakkar AK, et al. Dabigatran versus warfarin in the treatment of acute venous thromboembolism. N Engl J Med 2009;361:2342-52.

19. Schulman S, Kakkar AK, Goldhaber SZ, et al. Treatment of acute venous thromboembolism with dabigatran or warfarin and pooled analysis. Circulation 2014;129:764-72.

20. Agnelli G, Buller HR, Cohen A, et al. Oral apixaban for the treatment of acute venous thromboembolism. N Engl J Med 2013; 369:799-808.

21. Hokusai-VTE Investigators, Buller HR, Decousus H, et al. Edoxaban versus warfarin for the treatment of symptomatic venous thromboembolism. N Engl J Med 2013;369:1406-15.

22. McCaig LF, McLemore T. Plan and operation of the National Hospital Ambulatory Medical Survey. Series 1: programs and collection procedures. Vital Health Stat 1 1994;34:1-78.

23. Niska R, Bhuiya F, Xu J. National Hospital Ambulatory Medical Care Survey: 2007 emergency department summary. Natl Health Stat Report 2010;26:1-31.

24. Baglin T. Fifty per cent of patients with pulmonary embolism can be treated as outpatients. J Thromb Haemost 2010;8:2404-5.

25. Pollack CV, Schreiber D, Goldhaber SZ, et al. Clinical characteristics, management, and outcomes of patients diagnosed with acute pulmonary embolism in the emergency department: initial report of EMPEROR (Multicenter Emergency Medicine Pulmonary Embolism in the Real World Registry). J Am Coll Cardiol 2011;57:700-6. 